\title{
Effect of patient characteristics on the yield of prolonged baseline head-up tilt testing and the additional yield of drug provocation
}

\author{
A P Fitzpatrick, R J Lee, L M Epstein, M D Lesh, S Eisenberg, M M Scheinman
}

\begin{abstract}
Objective-To define the value of tilt testing and the additional yield of drug provocation over prolonged baseline tilt in different patient subgroups. (Many different protocols are in use for head-up tilt testing in heterogeneous groups of patients. Not all patients in reported series have recurrent syncope, and there is often a wide age range and a variable incidence of structural heart disease.)

Design-In a prospective study, baseline $60^{\circ}$ head-up tilt testing was undertaken for 45 minutes, initially without drug provocation. Patients who remained symptom free were given intravenous isoprenaline (isoproterenol) and further tilting or edrophonium (10 $\mathrm{mg}$ bolus) during tilt, in an order determined randomly before the start of the test. If they were symptom free after the first drug, they were given the other drug. A positive test was recorded when syncope or pre-syncope occurred with a rapid fall $(>30 \%)$ in blood pressure. The impact on tilt result of the type of symptoms, presence of significant structural heart disease (SHD), presence of a non-cardiovascular cause of sudden diminished consciousness (SDC), and age was then assessed by subgroup analysis.
\end{abstract}

Patients-145 patients (73 female, mean age 51 (25), range 8-94) with one or more episodes of pre-syncope or syncope.

Results -39 patients $(27 \%, 21$ female, age 49 (25) years) had positive tests and 106 (73\%, 52 female, age 52 (25) years) negative tests. $27(69 \%)$ had a positive test during baseline tilt at $20.5(10 \cdot 8)$ minutes, five (13\%) with isoprenaline infusion, and seven (18\%) with edrophonium bolus.

Patients with recurrent syncope rather than single syncopal episodes or single or recurrent pre-syncope were more likely to have a positive tilt test $(41 \% v 17 \%, P<$ 0.005 ) and patients with SHD or SDC (69/14 patients) were much less likely than patients without $(16 \% v 42 \%, P<0.0001)$. The yield of positive tests was similar if patients were below (26\%) or above $(27 \%)$ the mean age (50 years). When multiple factors were combined, the yield ranged from $0 \%$ for 21 patients under 50 years with SHD or SDC and without recurrent syncope to $73 \%$ in 11 patients over 50 years with recurrent syncope and no SHD or SDC. The additional yield in sub- groups over 45 minute baseline tilt (70 (11)\%) of isoprenaline (13 (10)\%) was similar to that of edrophonium (17 (8)\%, $P=$ NS), but six (50\% of those who were drug positive) patients required a second drug to produce a positive result (two with isoprenaline second, four with edrophonium second).

Conclusions-Head-up tilt testing in a heterogeneous population has a low yield. Simple clinical characteristics define the type of patient who is likely to have a positive tilt test and the patient who is not and in whom other investigations should receive priority. The great majority of positive tests will occur during prolonged baseline testing if this is used. Isoprenaline and edrophonium produced similar additional yields of positive tests.

(Heart 1996;76:406-411)

Keywords: head-up tilt; syncope; drug provocation

In recent years there has been a rapid growth in interest in reproducing symptoms during head-up tilt testing in patients with unexplained syncope, ${ }^{1-6}$ and thereby diagnosing vasovagal syncope or neurally mediated syncope. Because of the wide interest and rapid uptake of the technique many different protocols have developed in parallel, and many reported series have included heterogeneous patient groups. Some series have included patients with a wide age range, ${ }^{2}$ others have included patients with a history of pre-syncope rather than syncope. ${ }^{3}$ In other reports data have been reported only for patients with recurrent syncope, in a relatively narrow age band, and with a low rate of structural heart disease. ${ }^{1}$ In addition many different provocative agents have been used, ${ }^{278}$ and in most cases have been shown to have an acceptable specificity when tested in control groups. However, it is uncertain whether these agents increase sensitivity, because of the lack of an objective reference standard test for diagnosing vasovagal syncope.

A wide diversity of tilt protocols and patient types has led to uncertainty in comparing the results of different series and interpreting the results of clinical studies. We hypothesised that important clinical variables that might have an impact on tilt result included isolated syncope or isolated or recurrent pre-syncope 
only (rather than recurrent syncope), patient age, presence or absence of significant structural heart disease, and presence or absence of other likely causes of suddenly reduced consciousness. We also hypothesised that different tilt protocols might lead to very different tilt test results. Tilt variables that required further investigation include the duration of baseline tilting that should be used, the additional yield and impact on specificity of drug provocation, and a direct comparison of provocative agents. This prospective study was performed to attempt to address some of these issues.

\section{Patients and methods PATIENTS}

In a prospective fashion, all patients referred for tilting between March 1992 and December 1994 were entered into the study unless it was thought that there was a significant risk to the patient from administration of isoprenaline (isoproterenol) or edrophonium. Patients had either recurrent syncope or an isolated episode of syncope, or recurrent pre-syncope or an isolated episode of pre-syncope. Any patient referred for tilting, irrespective of clinical presentation, age, or the presence of significant structural heart disease disease was entered into the study, unless there was thought to be a contraindication to administration of one of the drugs. Significant structural heart disease (SHD) encompassed any documented cardiac condition in which an abnormality was present that might have given rise to diminished consciousness as a result of a bradyarrhythmia or a ventricular tachyarrhythmia. Any significant non-cardiovascular abnormality that might give rise to sudden diminished consciousness (SDC), such as a significant level of anaemia, electrolyte imbalance, endocrine imbalance, intracranial pathology, known seizure disorder, respiratory disorder, or substance abuse, was also noted.

\section{TILT TESTING AND DRUG PROVOCATION}

All patients were to undergo a 45 minute baseline tilt at $60^{\circ}$ after a 15 minute period resting supine. Patients were randomly assigned before the onset of tilt testing to receive isoprenaline (by supine graded intravenous infusion at increments of $0.5 \mu \mathrm{g} / \mathrm{min}$ in a dose sufficient to raise baseline supine heart rate by $20-25 \%$ followed by tilting to $60^{\circ}$ ) or edrophonium by $10 \mathrm{mg}$ bolus intravenous injection in the tilted position immediately after baseline tilt, on an intention to treat basis, if they remained asymptomatic after baseline tilt. If any patient remained asymptomatic after the first drug, they then received the other drug. The test was regarded as positive if at any stage patients failed to complete the 45 minute baseline tilt and both drug provocations without developing syncope or pre-syncopal symptoms and a clearly associated rapid (that is, occurring over $<1$ minute, but not immediately upon tilting) fall in mean arterial pressure (MABP) of $>30 \%$ over baseline (defined as peak MABP during $60^{\circ}$ tilt before any symptoms) blood pressure levels. The test was regarded as negative if they completed the whole sequence without symptoms. Patients were tilted in the morning or early afternoon after fasting overnight, and had an intravenous cannula inserted into a forearm vein at least 30 minutes before the start of the test. The electrocardiogram (ECG) was monitored continuously during the test and systolic, diastolic, and mean blood pressure were measured using an Criticon Dinomapp automatic sphygmomanometer every five minutes during asymptomatic tilt and continuously during symptoms and after edrophonium injection.

\section{STATISTICAL ANALYSIS}

A chi-square test was used to compare categoric data between subgroups.

\section{Results}

PATIENT CHARACTERISTICS (TABLE)

One hundred and forty five patients were randomised. Five patients were excluded from enrolment and analysis because the supervising physician thought that isoprenaline infusion represented an unwarranted risk because of the presence of significant structural heart disease (SHD). Seventy three patients $(50 \cdot 3 \%$, age 49 (25) years) were female and 72 $(49.7 \%$, age 53 (24) years) were male. Significant SHD or SDC was present in 83 out of 145 patients (39 female, age 57 (24) years). Sixty nine patients had SHD, with coronary artery disease and a remote myocardial infarction in 14 patients, chronic hypertension and documented left ventricular hypertrophy in 18 patients, valvar heart disease with secondary ventricular dysfunction in 11 patients, chronic hypertension and heart failure in eight patients, congenital heart disease in seven patients, documented conduction tissue disease in six patients (atrioventricular block in three, sinoatrial disease in three, none paced at the time of the tilt

Nature of underlying structural heart disease (SHD) (69 patients) and attributable cause of sudden diminished level of consciousness (SDC) (14 patients) in 83 patients

\begin{tabular}{lc}
\hline Condition & Patients \\
\hline SHD: & \\
CAD+MI & 14 \\
BP+LVH & 18 \\
VD+LV dysfunction & 11 \\
BP+LVF & 8 \\
CHD & 7 \\
CTD & 6 \\
OCT & 3 \\
Long Q-T & 1 \\
HCM & 1 \\
Total & 69 \\
SD: & 3 \\
Anaemia & 2 \\
Parkinson's Disease & 1 \\
Meningioma & 1 \\
Seizure disorder & 1 \\
Cocaine abuse & 1 \\
Hyponatraemia & 2 \\
Hypothyroidism & 3 \\
Severe obstructive airways disease & 14 \\
Total &
\end{tabular}

$\mathrm{CAD}+\mathrm{MI}$, coronary artery disease and a remote myocardial infarction; $\mathrm{BP}+\mathrm{LVH}$, chronic hypertension and documented left ventricular hypertrophy; $\mathrm{VD}+\mathrm{LV}$ dysfunction, valvar heart disease with secondary ventricular dysfunction; $\mathrm{BP}+\mathrm{LVF}$, chronic hypertension and heart failure; CHD, congenital heart disease; CTD, conduction tissue disease; OCT, orthotopic heart transplantation; HCM, hypertrophic cardiomyopathy. 
Results of tilting in all patients and in 20 different subgroups defined by a combination of clinical characteristics: $<50$ years or $>50$ years, patients below or above the mean age of the whole group; SHD/SDC, patients with significant structural heart disease or other likely cause for diminished level of consciousness; $<2$ syncope, patients with a single syncopal episode and/or single or multiple episodes of pre-syncope without frank loss of consciousness; $2+$ syncope, patients with recurrent syncope.

\section{$\%$ Negative $\square$ Edrophonium \% positive $\square$ Isoprenaline \% positive $\square$ Baseline \% positive}

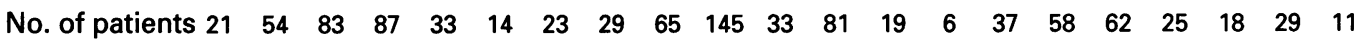

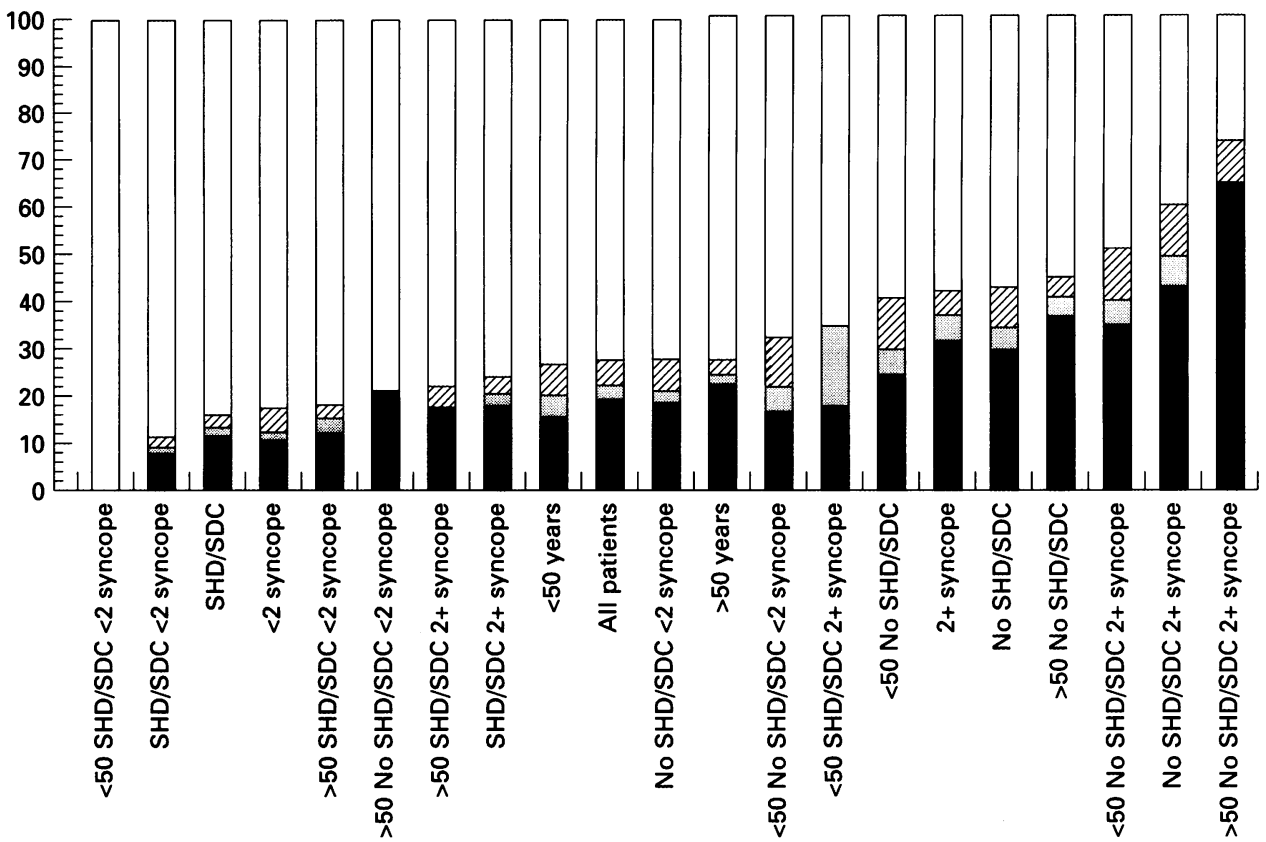

study), and orthotopic heart transplantation in three patients. One patient had hypertrophic cardiomyopathy and one had long-QT syndrome. Fourteen patients had SDC, including significant anaemia (haemoglobin concentration $<9 \mathrm{~g} / \mathrm{dl}$ ) in three, severe Parkinson's disease in two, a meningioma in one patient, a seizure disorder in one patient, hyponatraemia (plasma sodium concentration $<120 \mathrm{mEq} / \mathrm{l}$ ) in one patient, hypothyroidism in two patients, severe obstructive airways disease in three patients, and cocaine abuse in one patient. Sixty two patients had no SHD or SDC.

\section{TILT RESULTS (FIGURE)}

Role of patient characteristics

In the whole group 39 patients $(27 \%, 21$ female, age 49 (25) years) had positive and 106 (73\%, 52 female, age 52 (25) years) negative tilt tests. Twenty seven positive tests $(69 \%)$ occurred during baseline tilt at 20.5 $(10 \cdot 8)$ minutes, five $(13 \%)$ with isoprenaline infusion, and seven (18\%) with edrophonium bolus.

Fifty eight patients who had presented with recurrent syncope, rather than single syncopal episodes or single or recurrent pre-syncope, had a much higher yield from tilt testing (41\% $v 17 \%, \mathrm{P}<0.005)$. In the 83 patients with SHD or SDC there was a much lower yield than in the 62 patients without $(16 \% v 42 \%$, $P<0.0001)$. The yield was similar in the 65 patients below, as compared with the 80 patients above, the mean age $(26 \% v 27 \%, \mathrm{P}$ $=$ NS).

When multiple variables were combined a much greater discrepancy in the yield of tilttesting became apparent between subgroups. In patients without SHD or SDC those with recurrent syncope (29 patients) had a much higher yield $(59 \%)$ than those with only single syncopal episodes or only pre-syncope (33 patients, $27 \%$ positive, $P<0.03$ ). In patients with SHD or SDC there was no significant increase in yield if patients complained of recurrent syncope (seven of 29 patients, 24\%) than if they did not, (six of 54 patients, $11 \%$, $P=N S$ ). In patients over the mean age with SHD or SDC, recurrence of syncope rather than single episodes or pre-syncope alone made no difference to yield. Five of 23 patients over 50 years (22\%) with SHD or SDC and recurrent syncope had a positive response, as did six of $33(18 \%)$ without recurrent syncope. This yield was similar to that in 14 patients over 50 without structural heart disease and without recurrent syncope (three of 14, 21\%). There was no difference in yield between patients with recurrent syncope and no SHD or SDC under 50 years (nine of $18,50 \%$ ) and those over 50 years (eight of 11 , $73 \%, \mathrm{P}=\mathrm{NS}$ ).

The highest yield of all was seen in patients over 50 years without SHD or SDC presenting with recurrent syncope, with eight of 11 patients $(73 \%)$ having positive tests. This was significantly higher than in patients over 50 without SHD or SDC but without recurrent syncope (three of $14(21 \%), P<0.01)$, patients over 50 with recurrent syncope but with SHD or SDC (five of 23 (22\%), $P<0.005)$, and patients over 50 with SHD or SDC but without recurrent syncope, (six of 33 (18\%), $\mathrm{P}<0.0001)$

Among younger patients below 50 years, the highest yield was seen in 18 patients with recurrent syncope and no SHD or SDC (nine of $18(50 \%)$ ). The lowest yield of all was in 21 patients below 50 years with SHD or SDC, in whom there were no positive responses. The percentage yield of tilt table testing in all patients and in 20 subgroups is shown in the figure. 
THE YIELD OF BASELINE TILT AND ADDITIVE EFFECT OF DRUG PROVOCATION

Before tilt testing, 72 patients were randomised to receive isoprenaline first and 73 edrophonium first (if the test was still negative after the baseline tilt period of 45 minutes). In all 145 patients 27 of $39(69 \%)$ positive responses occurred during a 45 minute baseline tilt, at 21 (11) (range 3-45) minutes. Five patients had baseline, non-drug provoked, positive tests within 10 minutes (18.5\%), 10 (37\%) within 20 minutes, $20(72 \%)$ within 30 minutes, 26 (96\%) within 40 minutes, and 27 at 45 minutes.

Twelve patients had positive tilts during drug provocation after a negative 45 minute baseline tilt: five during isoprenaline infusion and seven with edrophonium injection. Of these, two were isoprenaline positive after negative responses to edrophonium injection and four were edrophonium positive after negative responses to isoprenaline infusion. Among the 20 subgroups of patients identified by clinical characteristics (figure), the additional yield of isoprenaline $(13(10) \%)$ and edrophonium (17(8)\%, $\mathrm{P}=\mathrm{NS}$ ), over 45 minute baseline tilt $(70(11) \%$ ) remained fairly constant in all the subgroups.

\section{Discussion}

This study shows that the yield of tilt table testing is greatly influenced by the clinical characteristics of the patients being tested. Vasovagal or neurally mediated syncope is apparently most likely to be elicited by tilting if a patient is over 50 years, has no significant structural heart disease or other likely cause for a sudden diminished level of consciousness, and has recurrent syncope. In contrast, no yield was obtained from tilt testing in patients under 50 years with single or no episodes of frank syncope in the presence of significant structural heart disease or another likely cause for the symptoms. A spectrum of likelihood of achieving a positive result, based on combinations of different clinical features, was found (figure). Furthermore, this study shows that the great majority of positive tests, independent of the subgrouping of patients, are obtained during prolonged baseline, unmedicated tilt, if this is used. An additional yield is obtained from both isoprenaline and edrophonium challenge. This yield was also fairly consistent in different subgroups.

The implications for patient selection for head-up tilt are significant. Tilting patients with isolated or recurrent pre-syncope or a single episode of syncope will lead to a reduced overall yield from the test. This may give the impression that tilt-table testing is less useful for patients with recurrent syncope than is actually the case. Tilt testing a heterogeneous population may result in an increased consumption of resources, particularly technical and physician time, and a diminished yield. Tilting only those with recurrent syncope aged over 50 years without an obvious or likely cause for symptoms will give the highest yield, but may lead to some patients remaining undi- agnosed. Such patients might benefit from the reassurance of being diagnosed, or from medical treatment. Conversely, investigating patients under 50 years without recurrent syncope but with structural heart disease initially by tilt testing is not justified on the basis of these results. Such an approach may even be hazardous to the patient. A positive test is much more likely statistically to be a false positive. Other tests much more likely to yield true positive results, such as electrophysiological testing, may be neglected, resulting in a misdiagnosis and inappropriate management.

These findings also help to explain the wide range of yields of tilt testing in published reports. As many as $75 \%^{1}$ and as few as $24 \%{ }^{9}$ of adult patients with recurrent syncope have been shown to have symptoms reproduced during head-up tilting. Until now one conclusion that could be drawn was that the results of this test were not reproducible between centres. However, this study suggests that one of the likely reasons is a difference in the type of patients being tested.

Another important cause of confusion over the utility of head-up tilt testing is the large number of different protocols in use. This has been the result of a rapid growth in interest, and no clear standardisation. Variables include the angle of tilt $\left(60-90^{\circ}{ }^{10}\right)$, the duration of baseline tilt, $\left(10-60\right.$ minutes $\left.^{211}\right)$, the use of vascular instrumentation (none, ${ }^{1}$ venous and arterial cannulation ${ }^{2}$ ), ' the use of drug provocation (none, ${ }^{1}$ isoprenaline, ${ }^{245}$ glyceryl trinitrate, ${ }^{7}$ edrophonium ${ }^{8}$ ). It has been shown by direct comparison that an angle of tilt less than $60^{\circ}$ reduces yield, ${ }^{12}$ but not that a greater angle increases it or leaves it unchanged. A number of different tilt angles have been reported, however, ${ }^{1} 1013$ making comparison difficult. This study suggests that if prolonged baseline $60^{\circ}$ tilting is used, the great majority of positive responses will occur without the need for drug provocation. This is likely to be because patients who would have had a positive response during drug administration with a shorter period of baseline tilt do so with protracted baseline tilt, shifting yield from one phase of the study to another. Analysis of the time of tilt-positivity during the baseline period shows a fairly even distribution of positive tests for every 10 minute period up to 40 minutes. The largest yield was between 20 and 29 minutes ( 10 of $27,37 \%$ ), which encompasses the mean value of time to syncope in this study $(20.5(10.8)$ minutes) and that of another (25 (10) minutes), ${ }^{12}$ on the basis of which we chose a baseline tilt period of 45 minutes (mean +2 standard deviations). ${ }^{12}$

An important question for the busy clinician is whether early drug provocation will maintain or possibly increase the yield of tilting, without loss of specificity, while shortening the test. Most baseline positives can be expected at around the 20th minute of $60^{\circ}$ tilt, ${ }^{12}$ however, and the time taken for many complete isoprenaline protocols can be considerable. Titrating heart rate during isoprenaline infusion with the patient returned to the 
supine position typically takes $10-15$ minutes. Even if the patient is immediately syncopal upon returning to $60^{\circ}$, the maximum time saving over 45 minute baseline tilt is only $10 \mathrm{~min}$ utes. If the test is not immediately positive, some protocols require a patient to undergo a graded infusion of isoprenaline up to $5 \mu \mathrm{g} / \mathrm{min},{ }^{413}$ with each graded increase followed by re-tilting to $60^{\circ}$, punctuated by a period of further infusion in the supine position. Consequently, up to two hours are required for a negative test.

The specificity of such protocols is also a cause of concern. Some studies have indicated that the false positive response rate of isoprenaline-tilt in control populations may be as high as $40-50 \% .{ }^{13}{ }^{14}$ In the case of administration of an edrophonium bolus during tilt, this study shows promise, in that the yield of tilting is apparently increased to a similar extent as with isoprenaline, but prolonged titration and resetting of the tilt table are not needed. However, caution is required since there are presently only limited data available on the specificity of edrophonium based on its use in control subjects. In the study by Lurie et $a l^{8}$ from UCSF, however, only one of 20 control subjects had a positive response with edrophonium challenge. Of concern in this study, are the $50 \%$ of patients who had positive tilt table tests with the second drug in the drug provocation sequence. In two cases the test was positive with isoprenaline after administration of edrophonium had produced no effect, and in four cases the test was positive with administration of edrophonium after isoprenaline failed to result in a positive test. There is a high likelihood of a increased number of false positive results after prolonged baseline tilt and the administration of two provocative agents.

Intravascular instrumentation has been shown to increase greatly the number of syncopal responses to orthostatic stress, particularly in young subjects. ${ }^{15}$ There are currently no data on the impact of venous and/or arterial cannulation on the result of tilt table testing in patients with syncope. However, all drug provocation protocols require venous cannulation and any increase in sensitivity may be at the expense of specificity, with some false positive results being due to the effect of cannulation of a vein. This risk may be avoided by using prolonged baseline tilt without venous cannulation. However, one of the continuing problems of the use of tilt-table testing to diagnose vasovagal or neurally mediated syncope in patients with unexplained syncope continues to be the lack of a gold standard test for the diagnosis with which to compare the results of tilting with different protocols to assess their relative sensitivity. Prolonged baseline tilt may be more specific than drugprovoked tilt, but the number of false negative tests that may occur by adopting baseline tilt alone remains unknown. Fortunately, the specificity of prolonged baseline tilt is known, however, with about $10 \%$ of control subjects experiencing a positive response to 45 minute tilting at $60^{\circ} .12$
THE IMPORTANCE OF CLINICAL EVALUATION

Based on the findings of this study, a clinical algorithm can be devised for the management of patients with syncope or pre-syncope. While all patients require a thorough clinical evaluation, the need for and type and sequence of test performed can be modified in the light of these findings. Patients with recurrent syncope, no structural heart disease, or other likely cause of syncope, particularly in older age groups when structural heart disease is likely to be commoner, should be selected for tilt-table testing at an early stage. In contrast, patients with single syncopal episodes or no frank syncope and with structural heart disease are very unlikely to have a positive tilt and may be best managed by event monitoring or electrophysiological study at an early stage. Such an approach may become increasingly attractive, and possibly mandated, in a new healthcare environment that stresses cost-control and cost-effectiveness in diagnosis and treatment, with critical pathways of care being defined for syncope and other syndromes.

IMPLICATIONS FOR TILT TABLE PROTOCOLS

In patients undergoing tilt table testing prolonged baseline $60^{\circ}$ tilt has a very high yield, and a high specificity, albeit with a presently unknown sensitivity, and should become standard practice. If $\mathbf{4 5}$ minute baseline tilt is negative, drug provocation may be used, but it should be appreciated that additional yield gained may be at the expense of specificity. Edrophonium may be superior to isoprenaline for use as a provocative agent. However, in this study, in $50 \%$ of edrophonium positive cases a bolus administration of the drug during tilt resulted in a positive test when both prolonged baseline tilt and isoprenaline challenge had been negative, raising questions about its specificity. Furthermore, there are few available data to assess the specificity of edrophonium, and it cannot be recommended in place of isoprenaline until its specificity is better understood.

\section{CONCLUSIONS}

This study shows that the clinical characteristics of patients with unexplained syncope or pre-syncope are critically important in determining the result of tilt table testing. Prolonged baseline tilt testing has a high yield with an additional much smaller yield from drug provocation. More data are needed to assess whether this additional yield is gained at the expense of specificity.

1 Fitzpatrick AP, Sutton R. Tilting towards a diagnosis in unexplained recurrent syncope. Lancet 1989;i:658-60

2 Almquist A, Goldenberg IF, Milstein S, Chen M-Y, Chen $\mathrm{X}$, Hansen $\mathrm{R}$, et al. Provocation of bradycardia and hypotension by isoproterenol infusion and upright posture in patients with unexplained syncope. $N$ Engl $\mathcal{F} M e d$ 1989;320:346-51.

3 Abi-Samra F, Maloney JD, Fouad-Tarazi FM, Castle LW. The usefulness of head-up tilt testing and haemodynamic investigations in the workup of syncope of unknown origin. PACE 1988;11:1202-14.

4 Sheldon R, Killam S. Methodology of isoproterenol-tilt table testing in patients with syncope. $7 \mathrm{Am}$ Coll Cardiol 1992;19:773-9.

5 Waxman MB, Yao L, Cameron DA, Wald RW, Roseman J. Isoproterenol induction of vasodepressor-type reaction in vasodepressor-prone persons. Am f Cardiol 1989;63: 58-65. 
6 Raviele A, Gasparini G, Di Pede F, Delise P, Bonso A, Piccolo E. Usefulness of head-up tilt test in evaluating patients with syncope of unknown origin and negative patients with syncope of unknown origin and negative

7 Raviele A, Gasparini G, Di Pede F, Menozzi C, Brignole $M$, Dinelli $M$, et al. Nitroglycerin infusion during upright tilt: a new test for the diagnosis of vasovagal syncope. $\mathrm{Am}$ Heart $\mathcal{~ 1 9 9 4 ; 1 2 7 : 1 0 3 - 1 1 . ~}$

8 Lurie KG, Dutton J, Mangat R, Newman D, Eisenberg S, Scheinman MM. Evaluation of edrophonium as a provocative agent for vasovagal syncope during head-up tilt-table testing. Am 7 Cardiol 1993;72:1286-90.

9 Grubb BP, Temesy-Armos P, Hahn H, Elliott L. Utility of tilt-table testing in th evaluation and management of syntilt-table testing in th evaluation and management

10 Pongiglione G, Fish FA, Strasburger JF, Benson DW Jr. Heart rate and blood pressure response to upright tilt in young patients with unexplained syncope. F $\mathrm{Am}$ Coll Cardiol 1990;16:165-70.

11 Kenny RA, Ingram A, Bayliss J, Sutton R. Head-up tilt: a useful test for the investigation of unexplained syncope. Lancet 1986;i:1352-4.

12 Fitzpatrick AP, Theodorakis G, Vardas P, Sutton R. Methodology of head-up tilt testing in patients with unexplained syncope. F Am Coll Cardiol 1991;17:125-30.

13 Kapoor WN, Brant N. Evaluation of syncope by upright tilt testing with isoproterenol. A nonspecific test. Ann Intern Med 1992;116:358-63.

14 Sheldon R. Evaluation of a single-stage isoproterenol-tilt table test in patients with syncope. F Am Coll Cardiol 1993;22:114-8.

15 Stevens PM. Cardiovascular dynamics during orthostasis and the influence of intravascular instrumentation. $A m \mathcal{F}$ Cardiol 1966;17:211-8.

\title{
Coronary ostial occlusion: "Have you seen anything like this?”
}

\author{
Iftikhar Ahmed, M C J Sissons, David Hesketh Roberts
}

A 48 year old deaf and dumb woman presented with a 12 month history that strongly suggested angina with recent worsening of chest pain. Diagnostic angiography showed normal coronary arteries. The procedure was uneventful but one hour later the patient suffered a fatal cardiorespiratory arrest. Postmortem examination revealed only that both coronary ostia were occluded by recent platelet rich antemortem thrombus in the absence of any underlying significant intimal disease or any evidence of trauma to the deeper tissues (figure). No primary site of embolisation was demonstrated. Stains for fungi and bacteria, autoimmune screening, and serology for syphilis were negative.

Any explanation to account for the pathology requires consideration of the cause of chest pain. This patient may have had an intense vasospastic potential and shut down her arteries over the catheters, thus allowing thrombus to develop. According to this hypothesis spasm would have been the cause of her prior angina. Alternatively she may have had an intense thrombotic potential which became manifest when the endothelium was damaged by catheters in the coronary orifices. This does not, however, explain the previous angina.

\section{Department of} Cardiology, Victoria Hospital, Blackpool I Ahmed

\section{Department of} Pathology, Victoria Hospital, Blackpool M C J Sissons

Department of Cardiology, Victoria Hospital, Blackpool D H Roberts

Correspondence to: Dr D H Roberts, Victoria Hospital, Whinney Heys Road, Blackpool, Lancashire FY3 8NR.

Accepted for publication 29 April 1996

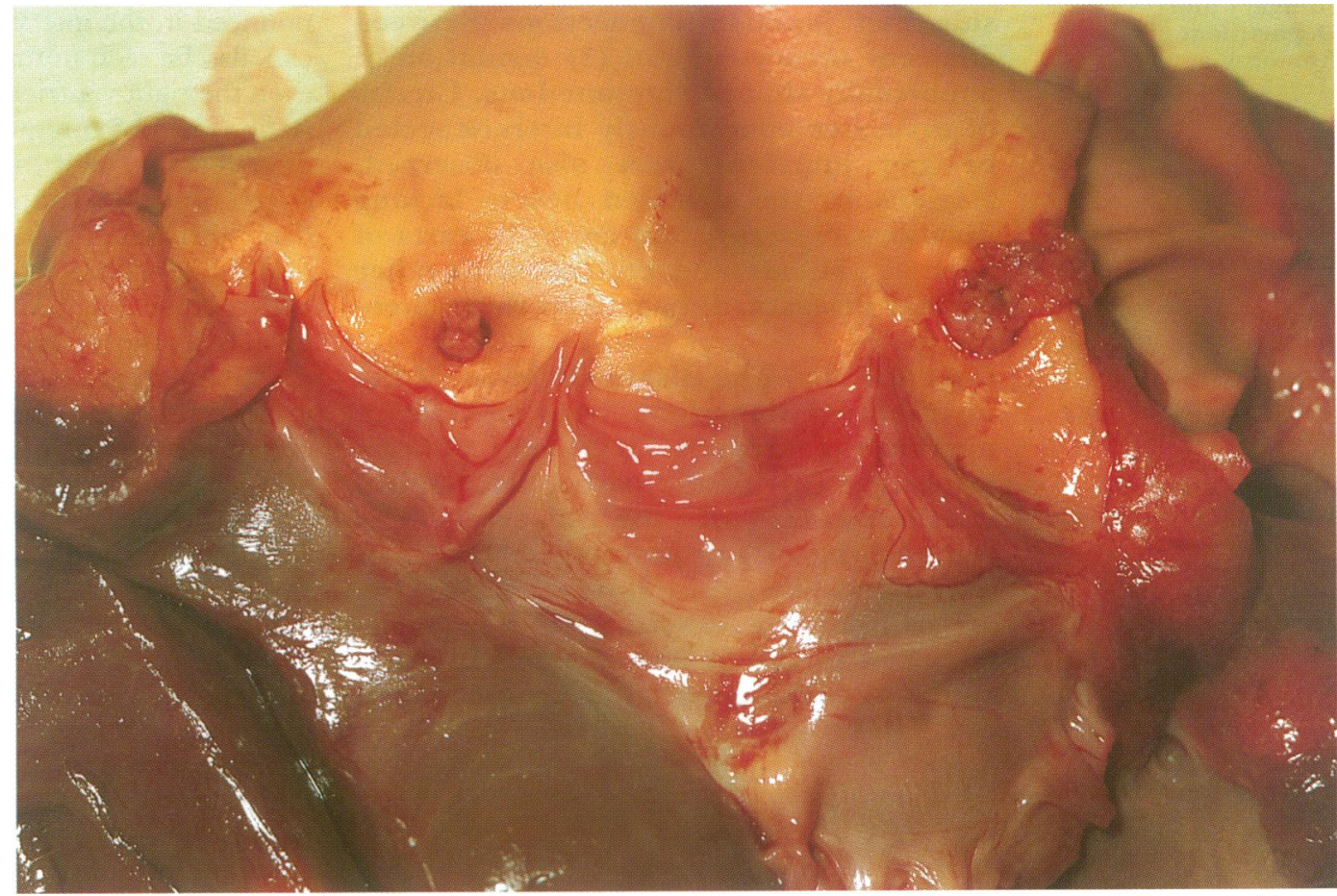

Anterior view of the ascending aorta showing thrombi at each coronary ostia. 